\title{
Preimage Entropy Dimension of Topological Dynamical Systems
}

\author{
Lei Liu, Xiaomin Zhou and Xiaoyao Zhou*
}

\begin{abstract}
We propose a new definition of preimage entropy dimension for continuous maps on compact metric spaces, investigate fundamental properties of the preimage entropy dimension, and compare the preimage entropy dimension with the topological entropy dimension. The defined preimage entropy dimension satisfies various basic properties of topological entropy dimension, for example, the preimage entropy dimension of a subsystem is bounded by that of the original system and topologically conjugated systems have the same preimage entropy dimension. Also, we discuss the relation between the preimage entropy dimension and the preimage entropy.
\end{abstract}

\section{Introduction}

In 1958, Kolmogorov applied the notion of entropy from information theory to ergodic theory. Since then, the concepts of entropy, in particular the topological entropy and measure theoretic entropy, were useful for studying topological and measure-theoretic structures of dynamical systems, that is, topological entropy (see [1,3,4,21]) and measuretheoretic entropy (see [10,18,34]). For instance, two conjugate systems have the same entropy and thus entropy is a numerical invariant of the class of conjugated dynamical systems. The theory of expansive dynamical systems has been closely related to the theory of topological entropy [5, 17, 33. Entropy and chaos are closely related, for example, a continuous map of interval is chaotic if and only if it has a positive topological entropy [2].

In [16], Hurley introduced several other entropy-like invariants for noninvertible maps. Nitecki and Przytycki 30] discussed preimage branch entropy (retaining Hurley's notation) according to the branches of the inverse map. Cheng and Newhouse [9] further extended the concept of topological entropy of a continuous map and gave the concept of pre-image entropy for compact dynamical systems. Several important preimage entropy invariants, such as pointwise preimage, pointwise branch entropy, partial preimage entropy, and bundle-like preimage entropy, etc., have been introduced and their relationships with

Received January 14, 2021; Accepted July 20, 2021.

Communicated by Cheng-Hsiung Hsu.

2020 Mathematics Subject Classification. 54H20, 37B20.

Key words and phrases. entropy dimension, preimage entropy, s-preimage entropy, preimage entropy dimension.

${ }^{*}$ Corresponding author. 
topological entropy have been established. E. Mihailescu and M. Urbanski [25 27] introduced and studied another related notion of preimage entropy, namely the inverse entropy and inverse pressure for noninvertible maps. The authors 8, 19, 23, 24 extended and studied as above some entropy-like invariants for the non-autonomous discrete dynamical systems given by a sequence of continuous self-maps of a compact topological space.

Although systems with positive entropy are much more complicated than those with zero entropy, zero entropy systems have various complexity, and have been studied by many authors (see $6,7,11,15,28,31]$ ). These authors adopted various methods to classify zero dynamical systems. Carvalho [6] introduced the notion of entropy dimension to distinguish the zero topological entropy systems and obtained some basic properties of entropy dimension. Cheng and Li $[7]$ further discussed entropy dimension of the probabilistic and the topological versions and constructed a symbolic subspace to achieve zero topological entropy and they have proved the symbolic subspace have full entropy dimension. Ferenczi and Park [13] investigated a new entropy-like invariant for the action of $\mathbb{Z}$ or $\mathbb{Z}^{d}$ on a probability space. Recently, Li, Wu and Zhu 22 investigated the pressure of a potential via the dynamics of preimage structure for noninvertible systems and considered the properties for the related equilibrium states.

In this paper we introduce the generalized $s$-preimage entropy and preimage dimension of a topological dynamical system, and study properties of the preimage entropy dimension. These properties include that the preimage entropy dimension of continuous maps on the finite spaces is zero, the preimage entropy dimension of a contractive continuous map is also zero and the preimage entropy dimension of a subsystem is bounded by that of the original system. We prove that the $s$-preimage entropy of $T^{n}$ is less than or equal to $n$ times the $s$-preimage entropy of $T$ and the preimage entropy dimension of $T^{n}$ equals the preimage entropy dimension of $T$. The main results show that the $s$-preimage entropy is an invariant, that is, topologically conjugate systems have the same $s$-preimage entropy and the $s$-preimage entropy of a direct product is the sum of the $s$-preimage entropies of the factors. Moreover, we discuss the relation between preimage entropy dimension and preimage entropy, and obtain that the preimage entropy dimension is less than or equal to one if the preimage entropy is less than infinity, the preimage entropy dimension is more than or equal to one if the preimage entropy equals infinity and the preimage entropy dimension equals one if the preimage entropy is larger than 0 and it is less than infinity.

\section{Preimage entropy dimension}

A topological dynamical system $(X, d, T)((X, T)$ for short) means that $(X, d)$ is a compact metric space together with a continuous self-map $T: X \rightarrow X$. Let $\mathbb{N}$ denote the set of all positive integers and let $\mathbb{N}^{*}=\mathbb{N} \cup\{0\}$. 
Let $n \in \mathbb{N}$. Define the metric $d_{T, n}$ on $X$ by

$$
d_{T, n}(x, y)=\max _{0 \leq j<n} d\left(T^{j}(x), T^{j}(y)\right)
$$

Given a subset $K \subseteq X$, a set $E \subseteq K$ is an $(n, \epsilon, K, T)$-separated set if, for any $x \neq y$ in $E$, one has $d_{T, n}(x, y)>\epsilon$. Let the quantity $r(n, \epsilon, K, T, d)(r(n, \epsilon, K, T)$ for short) be the maximal cardinality of $(n, \epsilon, K, T)$-separated subset of $K$. A subset $E$ is an $(n, \epsilon, K, T)$-spanning set if, for every $x \in K$, there is a $y \in E$ such that $d_{T, n}(x, y) \leq \epsilon$. Let $s(n, \epsilon, K, T, d)$ $(s(n, \epsilon, K, T)$ for short) be the minimal cardinality of any $(n, \epsilon, K, T)$-spanning set. It is standard that for any subset $K \subseteq X$,

$$
s(n, \epsilon, K, T) \leq r(n, \epsilon, K, T) \leq s(n, \epsilon / 2, K, T) .
$$

The topological entropy, $h_{\text {top }}(T)$ is given by

$$
h_{\mathrm{top}}(T)=\lim _{\epsilon \rightarrow 0} \limsup _{n \rightarrow \infty} \frac{1}{n} \log r(n, \epsilon, X, T) .
$$

Definition 2.1. 9] Let $(X, T)$ be a topological dynamical system. Let $\epsilon>0$ and $n \in \mathbb{N}$. Then

$$
h_{\text {pre }}(T)=\lim _{\epsilon \rightarrow 0} \limsup _{n \rightarrow \infty} \frac{1}{n} \log \sup _{x \in X, k \geq n} r\left(n, \epsilon, T^{-k}(x), T\right)
$$

is called the preimage entropy of $T$.

Note that $h_{\text {pre }}(T) \leq h_{\text {top }}(T)$, and if $T$ is a homeomorphism, then $h_{\text {pre }}(T)=0$.

Definition 2.2. [7] Let $(X, T)$ be a topological dynamical system and $s \in \mathbb{N}^{*}$. Then $s$-topological entropy of $T$ is defined as

$$
D(s, T)=\lim _{\epsilon \rightarrow 0} \limsup _{n \rightarrow \infty} \frac{1}{n^{s}} \log r(n, \epsilon, X, T)=\lim _{\epsilon \rightarrow 0} \limsup _{n \rightarrow \infty} \frac{1}{n^{s}} \log s(n, \epsilon, X, T) .
$$

In [6], the author proved that the $s$-topological entropy $D(s, T)$ shares the following property.

Proposition 2.3. (1) The map $s>0 \mapsto D(s, T)$ is positive and decreasing with $s$;

(2) There exists $s_{0} \in[0,+\infty]$, such that

$$
D(s, T)= \begin{cases}+\infty & \text { if } 0<s<s_{0}, \\ 0 & \text { if } s>s_{0}\end{cases}
$$

(3) $D\left(s, T^{m}\right) \leq m^{s} D(s, T)$. 
Proposition 2.3 (2) indicates that the value of $D(s, T)$ jumps from infinity to 0 at the two sides of some point $s_{0}$, which is similar to Hausdorff dimension. Analogous to the Hausdorff dimension, Cheng and Li $[7]$ defined the entropy dimension of $T$ as follows:

$$
D(T)=\sup \{s>0: D(s, T)=\infty\}=\inf \{s>0: D(s, T)=0\} .
$$

Now, analogous to entropy dimension, we begin our process to introduce our new definition of preimage entropy dimension.

Definition 2.4. Let $(X, d, T)$ be a topological dynamical system. Let $\epsilon>0, n \in \mathbb{N}$ and $s>0$. Then the $s$-preimage entropy of $T$ is defined as

$$
\begin{aligned}
h_{\text {pre }}^{d}(s, T) & =\lim _{\epsilon \rightarrow 0} \limsup _{n \rightarrow \infty} \frac{1}{n^{s}} \log \sup _{x \in X, k \geq n} r\left(n, \epsilon, T^{-k}(x), T\right) \\
& =\lim _{\epsilon \rightarrow 0} \limsup _{n \rightarrow \infty} \frac{1}{n^{s}} \log \sup _{x \in X, k \geq n} s\left(n, \epsilon, T^{-k}(x), T\right) .
\end{aligned}
$$

When there is no confusion, we use $h_{\text {pre }}(s, T)$ instead of $h_{\text {pre }}^{d}(s, T)$. Clearly, $h_{\text {pre }}(1, T)=$ $h_{\text {pre }}(T)$. If $T$ is a homeomorphism, then $h_{\text {pre }}(s, T)=0$. Let us prove that $h_{\text {pre }}(s, T)$ is independent of the metric on $X$.

Proposition 2.5. $h_{\mathrm{pre}}(s, T)$ is independent of the choice of metric on $X$.

Proof. Let $d_{1}$ and $d_{2}$ be two compatible metrics on $X$. Then, by the compactness of $X$, for every $\epsilon>0$, there is a $\delta>0$ such that, for all $x, y \in X$, if $d_{1}(x, y)<\delta$, then $d_{2}(x, y)<\epsilon$. It follows that $r\left(n, \epsilon, T^{-k}(x), T, d_{2}\right) \leq r\left(n, \delta, T^{-k}(x), T, d_{1}\right)$ for all $x \in X$ and for every $n \in \mathbb{N}$ with $k \geq n$. This shows that $h_{\text {pre }}^{d_{2}}(s, T) \leq h_{\text {pre }}^{d_{1}}(s, T)$. Interchanging $d_{1}$ and $d_{2}$, this gives the opposite inequality. Therefore, $h_{\text {pre }}^{d_{1}}(s, T)=h_{\text {pre }}^{d_{2}}(s, T)$.

We can define the preimage entropy dimension of $T$ as the following

$$
D_{\text {pre }}(T)=\inf \left\{s>0: h_{\text {pre }}(s, T)=0\right\}=\sup \left\{s>0: h_{\text {pre }}(s, T)=\infty\right\} .
$$

It is clear that $h_{\text {pre }}(s, T) \leq D(s, T)$, and that $D_{\text {pre }}(T) \leq D(T)$.

Proposition 2.6. If $X$ is a finite set, then $D_{\text {pre }}(T)=0$.

Proof. Let $\epsilon>0, n \in \mathbb{N}$ and $s>0$. Since $X$ is finite, $r\left(n, \epsilon, T^{-k}(x), T\right) \leq \operatorname{card}(X)$. Hence,

$$
\begin{aligned}
h_{\text {pre }}(s, T) & =\lim _{\epsilon \rightarrow 0} \limsup _{n \rightarrow \infty} \frac{1}{n^{s}} \log \sup _{x \in X, k \geq n} r\left(n, \epsilon, T^{-k}(x), T\right) \\
& \leq \lim _{\epsilon \rightarrow 0} \limsup _{n \rightarrow \infty} \frac{1}{n^{s}} \log \operatorname{card}(X)=0 .
\end{aligned}
$$

This shows that $h_{\text {pre }}(s, T)=0$ for any $s>0$. Therefore, $D_{\text {pre }}(T)=0$. 
We can easily obtain the following proposition by using the method of 6$]$.

Proposition 2.7. (1) The map $s>0 \mapsto h_{\text {pre }}(s, T)$ is positive and decreasing with $s$;

(2) There exists $s_{0} \in[0,+\infty]$, such that

$$
h_{\text {pre }}(s, T)= \begin{cases}+\infty & \text { if } 0<s<s_{0}, \\ 0 & \text { if } s>s_{0} .\end{cases}
$$

Proposition 2.8. If $T: X \rightarrow X$ is a contractive continuous map, then $D_{\text {pre }}(T)=0$.

Proof. If $T: X \rightarrow X$ is a contractive map, then $T$ diminishes distance. Then

$$
s\left(n, \epsilon, T^{-k}(x), T\right) \leq s\left(n-1, \epsilon, T^{-k}(x), T\right) \leq \cdots \leq s\left(1, \epsilon, T^{-k}(x), T\right)
$$

and so

$$
\begin{aligned}
h_{\text {pre }}(s, T) & =\lim _{\epsilon \rightarrow 0} \limsup _{n \rightarrow \infty} \frac{1}{n^{s}} \log \sup _{x \in X, k \geq n} s\left(n, \epsilon, T^{-k}(x), T\right) \\
& \leq \lim _{\epsilon \rightarrow 0} \limsup _{n \rightarrow \infty} \frac{1}{n^{s}} \log \sup _{x \in X, k \geq n} s\left(1, \epsilon, T^{-k}(x), T\right)=0 \quad \text { for all } s>0 .
\end{aligned}
$$

This shows that $h_{\text {pre }}(s, T)=0$ for all $s>0$. Therefore, $D_{\text {pre }}(T)=0$.

Denote by $K(X, T)$ the set of all $T$-invariant nonempty compact subsets of $X$, that is, $K(X, T)=\{F \subseteq X: F \neq \emptyset, F$ is compact and $T(F) \subseteq F\}$. Since $X$ is compact, it follows from $T(X) \subseteq X$ that $K(X, T) \neq \emptyset$.

Definition 2.9. Let $(X, T)$ be a topological dynamical system. Let $\epsilon>0$ and $n \in \mathbb{N}$. For $F \in K(X, T)$,

$$
h_{\mathrm{pre}}\left(s,\left.T\right|_{F}, F\right)=\lim _{\epsilon \rightarrow 0} \limsup _{n \rightarrow \infty} \frac{1}{n^{s}} \log \sup _{x \in F, k \geq n} r\left(n, \epsilon,\left(\left.T\right|_{F}\right)^{-k}(x),\left.T\right|_{F}\right)
$$

is called $s$-preimage entropy of $T$ on $F$, where $\left.T\right|_{F}: F \rightarrow F$ is the induced map of $T$, that is, for any $x \in F,\left.T\right|_{F}(x)=T(x)$. The preimage entropy dimension of $T$ restricted to $F$ is given by

$$
D_{\text {pre }}\left(\left.T\right|_{F}, F\right)=\inf \left\{s>0: h_{\text {pre }}\left(s,\left.T\right|_{F}, F\right)=0\right\}=\sup \left\{s>0: h_{\text {pre }}\left(s,\left.T\right|_{F}, F\right)=\infty\right\} .
$$

Proposition 2.10. If $F_{1}, F_{2} \in K(X, T)$ and $F_{1} \subseteq F_{2}$, then $D_{\text {pre }}\left(\left.T\right|_{F_{1}}, F_{1}\right) \leq D_{\text {pre }}\left(\left.T\right|_{F_{2}}, F_{2}\right)$.

Proof. Let $\epsilon>0$ and $n, k \in \mathbb{N}$ with $k \geq n$, and let $x \in F_{1}$ and $E \subseteq\left(\left.T\right|_{F_{1}}\right)^{-k}(x)$ be an $\left(n, \epsilon,\left(\left.T\right|_{F_{1}}\right)^{-k}(x),\left.T\right|_{F_{1}}\right)$-separated subset with the maximal cardinality. For $x \in F_{1}$, we have

$$
F_{2} \supseteq\left(\left.T\right|_{F_{2}}\right)^{-k}(x) \supseteq\left(\left.T\right|_{F_{1}}\right)^{-k}(x) \subseteq F_{1} \subseteq F_{2} .
$$


Hence, $E$ is an $\left(n, \epsilon,\left(\left.T\right|_{F_{2}}\right)^{-k}(x),\left.T\right|_{F_{2}}\right)$-separated subset of $\left(\left.T\right|_{F_{2}}\right)^{-k}(x)$. Therefore,

$$
r\left(n, \epsilon,\left(\left.T\right|_{F_{1}}\right)^{-k}(x),\left.T\right|_{F_{1}}\right) \leq r\left(n, \epsilon,\left(\left.T\right|_{F_{2}}\right)^{-k}(x),\left.T\right|_{F_{2}}\right) .
$$

Furthermore, we have

$$
\begin{aligned}
& \lim _{\epsilon \rightarrow 0} \limsup _{n \rightarrow \infty} \frac{1}{n^{s}} \log \sup _{x \in F_{1}, k \geq n} r\left(n, \epsilon,\left(\left.T\right|_{F_{1}}\right)^{-k}(x),\left.T\right|_{F_{1}}\right) \\
\leq & \lim _{\epsilon \rightarrow 0} \limsup _{n \rightarrow \infty} \frac{1}{n^{s}} \log \sup _{x \in F_{2}, k \geq n} r\left(n, \epsilon,\left(\left.T\right|_{F_{2}}\right)^{-k}(x),\left.T\right|_{F_{2}}\right) .
\end{aligned}
$$

This shows that $h_{\text {pre }}\left(s,\left.T\right|_{F_{1}}, F_{1}\right) \leq h_{\text {pre }}\left(s,\left.T\right|_{F_{2}}, F_{2}\right)$. By Definition 2.9, we have

$$
D_{\text {pre }}\left(\left.T\right|_{F_{1}}, F_{1}\right) \leq D_{\text {pre }}\left(\left.T\right|_{F_{2}}, F_{2}\right)
$$

Remark 2.11. Fix $s>0$. For $F_{i} \in K(X, T), i \in \mathbb{N}$, if $F=\bigcup_{i=1}^{m} F_{i}$ and $F \in K(X, T)$, then $h_{\text {pre }}\left(s,\left.T\right|_{F}, F\right)=\max _{1 \leq i \leq m} h_{\text {pre }}\left(s,\left.T\right|_{F_{i}}, F_{i}\right)$ and $D_{\text {pre }}\left(\left.T\right|_{F}, F\right) \leq D_{\text {pre }}(T, X)$.

Example 2.12. Let $\left(\Sigma_{2}, \sigma\right)$ be a one-sided symbolic dynamical system, where $\Sigma_{2}=\{x=$ $\left(x_{n}\right)_{n=0}^{\infty}: x_{n} \in\{0,1\}$ for every $\left.n\right\}, \sigma\left(x_{0}, x_{1}, x_{2}, \ldots\right)=\left(x_{1}, x_{2}, \ldots\right)$. Then $D_{\text {pre }}(\sigma)=1$.

Considering $\{0,1\}$ as a discrete space and putting product topology on $\Sigma_{2}$, an admissible metric $\rho$ on the space $\Sigma_{2}$ is defined by

$$
\rho(x, y)=\sum_{n=0}^{\infty} \frac{d\left(x_{n}, y_{n}\right)}{2^{n}},
$$

where

$$
d\left(x_{n}, y_{n}\right)= \begin{cases}0 & \text { if } x_{n}=y_{n} \\ 1 & \text { if } x_{n} \neq y_{n}\end{cases}
$$

for $x=\left(x_{0}, x_{1}, \ldots\right), y=\left(y_{0}, y_{1}, \ldots\right) \in \Sigma_{2}$. By Robinson [32], $\Sigma_{2}$ is a compact metric space.

From Nitecki [29] and Cheng-Newhouse [9], $h_{\text {pre }}(\sigma)=\log 2$. Since

$$
\begin{aligned}
& \lim _{\epsilon \rightarrow 0} \limsup _{n \rightarrow \infty} \frac{1}{n^{s}} \log \sup _{x \in \Sigma_{2}, k \geq n} r\left(n, \epsilon, \sigma^{-k}(x), \sigma\right) \\
= & \lim _{\epsilon \rightarrow 0} \limsup _{n \rightarrow \infty} \frac{1}{n} \log \sup _{x \in \Sigma_{2}, k \geq n} s\left(n, \epsilon, \sigma^{-k}(x), \sigma\right)\left(n^{1-s}\right)= \begin{cases}+\infty & \text { if } s<1, \\
\log 2 & \text { if } s=1, \\
0 & \text { if } s>1 .\end{cases}
\end{aligned}
$$

Therefore, $D_{\text {pre }}(\sigma)=1$.

Example 2.13. Let $T: K \rightarrow K$ is a homeomorphism of the unit circle $K$. Then the preimage entropy dimension $D_{\text {pre }}(T)=0$. 
Without loss of generality, suppose the circle has length 1. First, choose $\epsilon>0$ small enough such that if $d(x, y) \leq \epsilon$ for all $x, y \in K$, then $d\left(T^{-1} x, T^{-1} y\right) \leq 1 / 4$.

Using the definition of spanning sets for $T^{-k}(x)$, it is clear that

$$
r\left(1, \epsilon, T^{-k}(x), T\right) \leq r(1, \epsilon, K, T) \leq\left[\frac{1}{\epsilon}\right]+1,
$$

where $[1 / \epsilon]$ is integer part of $1 / \epsilon$.

We claim that $r\left(n, \epsilon, T^{-k}(x), T\right) \leq n([1 / \epsilon]+1)$. By Walters' book 35, $r(n, \epsilon, K, T) \leq$ $n([1 / \epsilon]+1)$. Moreover, $r\left(n, \epsilon, T^{-k}(x), T\right) \leq r(n, \epsilon, K, T)$. Furthermore, $r\left(n, \epsilon, T^{-k}(x), T\right) \leq$ $n([1 / \epsilon]+1)$. Therefore, for any positive value $s$,

$$
\lim _{\epsilon \rightarrow 0} \limsup _{n \rightarrow \infty} \frac{1}{n^{s}} \log \sup _{x \in X, k \geq n} r\left(n, \epsilon, T^{-k}(x), T\right) \leq \limsup _{n \rightarrow \infty} \frac{\log n([1 / \epsilon]+1)}{n^{s}}=0,
$$

which implies that $D_{\text {pre }}(T)=0$.

\section{Main results}

Theorem 3.1. Let $(X, T)$ be a topological dynamical system. Then

(1) if $s>0$ and $m \in \mathbb{N}$, then $h_{\text {pre }}\left(s, T^{m}\right) \leq m^{s} h_{\text {pre }}(s, T)$,

(2) if $0<s \leq 1$ and $m \in \mathbb{N}$, then $h_{\text {pre }}\left(s, T^{m}\right) \leq m h_{\text {pre }}(s, T)$,

(3) if $m \in \mathbb{N}$, then $D_{\text {pre }}\left(T^{m}\right)=D_{\text {pre }}(T)$.

Proof. (1) Given $s>0$ and $m \in \mathbb{N}$. Write $g=T^{m}$. Let $k \geq n$ and $x \in X$. It is clear that

$$
r\left(n, \epsilon, g^{-k}(x), g\right) \leq r\left(m n, \epsilon, T^{-m k}(x), T\right) .
$$

Hence, we have

$$
\begin{aligned}
& \limsup _{n \rightarrow \infty} \frac{1}{n^{s}} \log \sup _{x \in X, k \geq n} r\left(n, \epsilon, g^{-k}(x), g\right) \\
\leq & \limsup _{n \rightarrow \infty} \frac{1}{n^{s}} \log \sup _{x \in X, k \geq n} r\left(n m, \epsilon, T^{-m k}(x), T\right) \\
= & \limsup _{n \rightarrow \infty} \frac{m^{s}}{(n m)^{s}} \log \sup _{x \in X, k \geq n} r\left(n m, \epsilon, T^{-m k}(x), T\right) \\
\leq & \limsup _{n \rightarrow \infty} \frac{m^{s}}{(n m)^{s}} \log \sup _{x \in X, k \geq m n} r\left(n m, \epsilon, T^{-k}(x), T\right) \\
= & m^{s} \limsup _{n \rightarrow \infty} \frac{1}{(n m)^{s}} \log \sup _{x \in X, k \geq m n} r\left(n m, \epsilon, T^{-k}(x), T\right) \\
\leq & m^{s} \limsup _{n \rightarrow \infty} \frac{1}{(n m)^{s}} \log \sup _{x \in X, k \geq n} r\left(n m, \epsilon, T^{-k}(x), T\right) .
\end{aligned}
$$


Furthermore,

$$
\begin{aligned}
& \lim _{\epsilon \rightarrow 0} \limsup _{n \rightarrow \infty} \frac{1}{n^{s}} \log \sup _{x \in X, k \geq n} r\left(n, \epsilon, g^{-k}(x), g\right) \\
\leq & m^{s} \lim _{\epsilon \rightarrow 0} \limsup _{n \rightarrow \infty} \frac{1}{(n m)^{s}} \log \sup _{x \in X, k \geq n} r\left(n m, \epsilon, T^{-k}(x), T\right) .
\end{aligned}
$$

Therefore, $h_{\text {pre }}\left(s, T^{m}\right) \leq m^{s} h_{\text {pre }}(s, T)$.

(2) Since $m^{s} \leq m$ for $0<s \leq 1$ and $m \in \mathbb{N}$, it follows that $h_{\text {pre }}\left(s, T^{m}\right) \leq m h_{\text {pre }}(s, T)$ by the above result of (1).

(3) Suppose $h_{\text {pre }}(s, T)=0$ for some $s>0$. From the above (1), we have $h_{\text {pre }}\left(s, T^{m}\right)=0$ for every $m \in \mathbb{N}$. Hence, $\left\{s>0: h_{\text {pre }}(s, T)=0\right\} \subseteq\left\{s>0: h_{\text {pre }}\left(s, T^{m}\right)=0\right\}$, further, $\inf \left\{s>0: h_{\text {pre }}\left(s, T^{m}\right)=0\right\} \leq \inf \left\{s>0: h_{\text {pre }}(s, T)=0\right\}$. This shows that $D_{\text {pre }}\left(T^{m}\right) \leq D_{\text {pre }}(T)$. Next, we prove that $D_{\text {pre }}\left(T^{m}\right) \geq D_{\text {pre }}(T)$. Since

$$
h_{\text {pre }}(s, T)=\lim _{\epsilon \rightarrow 0} \limsup _{n \rightarrow \infty} \frac{1}{n^{s}} \log \sup _{x \in X, k \geq n} s\left(n, \epsilon, T^{-k}(x), T\right),
$$

then given $\alpha>0$, there exists $x_{0} \in X$ such that

$$
h_{\text {pre }}(s, T)-\alpha<\lim _{\epsilon \rightarrow 0} \limsup _{n \rightarrow \infty} \frac{1}{n^{s}} \log \sup _{k \geq n} s\left(n, \epsilon, T^{-k}\left(x_{0}\right), T\right) .
$$

Fix $m \in \mathbb{N}$, we have

$$
h_{\text {pre }}\left(s, T^{m}\right)=\lim _{\epsilon \rightarrow 0} \limsup _{n \rightarrow \infty} \frac{1}{n^{s}} \log \sup _{y \in X, k \geq n} s\left(n, \epsilon, T^{-m k}(y), T^{m}\right) .
$$

Taking $y=T^{m k-k}\left(x_{0}\right)$, we get

$$
h_{\text {pre }}\left(s, T^{m}\right) \geq \lim _{\epsilon \rightarrow 0} \limsup _{n \rightarrow \infty} \frac{1}{n^{s}} \log \sup _{k \geq n} s\left(n, \epsilon, T^{-k}\left(x_{0}\right), T^{m}\right) .
$$

As $X$ is a compact space, $T, T^{2}, \ldots, T^{m}$ are uniformly continuous on $X$, given $\epsilon>0$, there exists $0<\delta<\epsilon$ such that

$$
d(x, y)<\delta \quad \Longrightarrow \quad \max _{0 \leq i<m} d\left(T^{i}(x), T^{i}(y)\right)<\epsilon .
$$

Then we get $s\left(m n, \epsilon, T^{-k}\left(x_{0}\right), T\right) \leq s\left(n, \delta, T^{-k}\left(x_{0}\right), T^{m}\right)$. By (3.1) and (3.2), we have

$$
\begin{aligned}
h_{\text {pre }}\left(s, T^{m}\right) & \geq \lim _{\delta \rightarrow 0} \limsup _{n \rightarrow \infty} \frac{1}{n^{s}} \log \sup _{k \geq n} s\left(n, \delta, T^{-k}\left(x_{0}\right), T^{m}\right) \\
& \geq \lim _{\epsilon \rightarrow 0} \limsup _{n \rightarrow \infty} m^{s} \frac{1}{(m n)^{s}} \log \sup _{k \geq n} s\left(m n, \epsilon, T^{-k}\left(x_{0}\right), T\right) \\
& \geq m^{s}\left(h_{\text {pre }}(s, T)-\alpha\right) .
\end{aligned}
$$

Letting $\alpha \rightarrow 0$, this implies that $h_{\text {pre }}\left(s, T^{m}\right) \geq m^{s} h_{\text {pre }}(s, T)$ for all $s>0$. This shows that $D_{\text {pre }}\left(T^{m}\right) \geq D_{\text {pre }}(T)$. 
Theorem 3.2. Let $(X, T)$ be a topological dynamical system. Then

(1) if $h_{\text {pre }}(T)<+\infty$, then $D_{\text {pre }}(T) \leq 1$.

(2) if $0<h_{\text {pre }}(T)<+\infty$, then $D_{\text {pre }}(T)=1$.

Proof. (1) If $h_{\text {pre }}(T)<+\infty$, then

$$
\lim _{\epsilon \rightarrow 0} \limsup _{n \rightarrow \infty} \frac{1}{n} \log \sup _{x \in X, k \geq n} r\left(n, \epsilon, T^{-k}(x), T\right)<+\infty .
$$

Therefore, for all $s>1$, we have

$$
\begin{aligned}
& \lim _{\epsilon \rightarrow 0} \limsup _{n \rightarrow \infty} \frac{1}{n^{s}} \log \sup _{x \in X, k \geq n} r\left(n, \epsilon, T^{-k}(x), T\right) \\
= & \lim _{\epsilon \rightarrow 0} \limsup _{n \rightarrow \infty} \frac{1}{n^{s-1}} \cdot \frac{1}{n} \log \sup _{x \in X, k \geq n} r\left(n, \epsilon, T^{-k}(x), T\right) \\
= & \lim _{n \rightarrow \infty} \frac{1}{n^{s-1}} \lim _{\epsilon \rightarrow 0} \limsup _{n \rightarrow \infty} \frac{1}{n} \log \sup _{x \in X, k \geq n} r\left(n, \epsilon, T^{-k}(x), T\right) \\
= & 0 \cdot h_{\text {pre }}(T)=0 .
\end{aligned}
$$

Therefore, $D_{\text {pre }}(T) \leq 1$.

(2) If $h_{\text {pre }}(T)<+\infty$, then we get from (1) that $D_{\text {pre }}(T) \leq 1$. As $h_{\text {pre }}(T)>0$, it is at $s=1$ that the map $s \mapsto h_{\text {pre }}(s, T)$ changes its value:

$$
h_{\text {pre }}(s, T)= \begin{cases}+\infty & \text { if } s<1 \\ h_{\text {pre }}(T) & \text { if } s=1 \\ 0 & \text { if } s>1\end{cases}
$$

This means that $D_{\text {pre }}(T)=1$.

In 20], Kuang, Cheng, Ma and Li gave a formula for the lower entropy dimension in subshift symbolic space and proved that the topological entropy dimension is $\log 2$ in the subshift symbolic space if $s=1 / 2$. Now, we will prove that the preimage entropy dimension is also $\log 2$ in the same subshift symbolic space of 20 if $s=1 / 2$, which shows the preimage entropy dimension is nonzero but strictly less than 1 .

Example 3.3. Let $\left(\Sigma_{2}, \sigma\right)$ be a one-sided symbolic dynamical system in Example 2.12 . Let

$$
X_{1}=\left\{x=\left(x_{i}\right)_{i=0}^{\infty}: x_{i}=0 \text { for } i \neq p^{2}, p=1,2,3,4, \ldots\right\},
$$

$X_{k}=\sigma^{k-1}\left(X_{1}\right)$ for $k \in \mathbb{N}$ and $X=\overline{\bigcup_{k \in \mathbb{N}} X_{k}}$. Then $\left(X,\left.\sigma\right|_{X}\right)$ is a subshift of $\left(\Sigma_{2}, \sigma\right)$ and $h_{\text {pre }}\left(1 / 2,\left.\sigma\right|_{X}\right)=\log 2$, further, $D_{\text {pre }}\left(\left.\sigma\right|_{X}\right)=1 / 2$. 
From 20], $\left(X,\left.\sigma\right|_{X}\right)$ is a subshift of $\left(\Sigma_{2}, \sigma\right)$ and $D\left(1 / 2,\left.\sigma\right|_{X}\right)=\log 2$. Clearly, $h_{\text {pre }}(1 / 2$, $\left.\left.\sigma\right|_{X}\right) \leq D\left(1 / 2,\left.\sigma\right|_{X}\right)$, further, we have $h_{\text {pre }}\left(1 / 2,\left.\sigma\right|_{X}\right) \leq \log 2$. In the following, we will prove $h_{\text {pre }}\left(1 / 2,\left.\sigma\right|_{X}\right) \geq \log 2$. Take $x^{*}=\left(x_{i}^{*}\right)_{i=0}^{\infty} \in X$ such that

$$
x_{i}^{*}= \begin{cases}0 & \text { for } i \neq p^{2}, p=1,2,3, \ldots \\ 1 & \text { otherwise }\end{cases}
$$

Fixed $n \in \mathbb{N}$. We put $p \in \mathbb{N}$ such that $p^{2} \leq n<(p+1)^{2}$. Let $\epsilon>0$. Then there exists $N \in \mathbb{N}$ such that $2^{-(N+1)}<\epsilon \leq 2^{-N}$. Moreover, we take $k^{*}=(p+1)^{2}+N+2$, then $\sigma^{k^{*}}\left(x^{*}\right) \in X$. Hence, we have

$$
r\left(n, \epsilon,\left(\left.\sigma\right|_{X}\right)^{-k^{*}}\left(\sigma^{k^{*}}\left(x^{*}\right)\right),\left.\sigma\right|_{X}\right) \geq 2^{p}
$$

Furthermore, for $s=1 / 2$, we have

$$
\begin{aligned}
& \lim _{\epsilon \rightarrow 0} \limsup _{n \rightarrow \infty} \frac{1}{n^{1 / 2}} \log \sup _{x \in X, k \geq n} r\left(n, \epsilon,\left(\left.\sigma\right|_{X}\right)^{-k}(x),\left.\sigma\right|_{X}\right) \\
\geq & \lim _{\epsilon \rightarrow 0} \limsup _{n \rightarrow \infty} \frac{1}{n^{1 / 2}} \log r\left(n, \epsilon,\left(\left.\sigma\right|_{X}\right)^{-k^{*}}\left(\sigma^{k^{*}}\left(x^{*}\right)\right),\left.\sigma\right|_{X}\right) \\
\geq & \lim _{n \rightarrow \infty} \frac{1}{n^{1 / 2}} \log 2^{p}=\lim _{n \rightarrow \infty} \frac{p \log 2}{n^{1 / 2}}=\log 2 .
\end{aligned}
$$

This implies $h_{\text {pre }}\left(1 / 2,\left.\sigma\right|_{X}\right) \geq \log 2$. Therefore, we get $h_{\text {pre }}\left(1 / 2,\left.\sigma\right|_{X}\right)=\log 2$ and $D_{\text {pre }}\left(\left.\sigma\right|_{X}\right)$ $=1 / 2$.

Let $\left(X, T_{1}\right)$ and $\left(Y, T_{2}\right)$ be two topological dynamical systems. Then, $\left(X, T_{1}\right)$ is an extension of $\left(Y, T_{2}\right)$, or $\left(Y, T_{2}\right)$ is a factor of $\left(X, T_{1}\right)$ if there exists a surjective continuous map $\pi: X \rightarrow Y$ (called a factor map) such that $\pi \circ T_{1}(x)=T_{2} \circ \pi(x)$ for every $x \in X$. If $\pi$ is a homeomorphism, then $\left(X, T_{1}\right)$ and $\left(Y, T_{2}\right)$ are said to be topologically conjugate and the homeomorphism $\pi$ is called a conjugate map.

Theorem 3.4. Let $\left(X, d, T_{1}\right)$ and $\left(Y, d^{\prime}, T_{2}\right)$ be two topological dynamical systems. If $\left(Y, T_{2}\right)$ is a factor of $\left(X, T_{1}\right)$ with a factor map $\pi: X \rightarrow Y$, then $h_{\text {pre }}\left(s, T_{1}\right) \geq h_{\text {pre }}\left(s, T_{2}\right)$ for all $s>0$. Moreover, if $\left(X, T_{1}\right)$ and $\left(Y, T_{2}\right)$ are topologically conjugate with a conjugate map $\pi: X \rightarrow Y$, then $h_{\text {pre }}\left(s, T_{1}\right)=h_{\text {pre }}\left(s, T_{2}\right)$ for all $s>0$.

Proof. Let $n \in \mathbb{N}$ and $k \geq n$. Since $X$ is compact and $\pi$ is continuous, $\pi$ is uniform continuous. So for any given $\epsilon>0$, there exists $\delta>0$ such that $d\left(x_{1}, x_{2}\right) \geq \delta$ whenever $d^{\prime}\left(\pi\left(x_{1}\right), \pi\left(x_{2}\right)\right) \geq \epsilon$. Fix $y \in Y$, let $E\left(n, \epsilon, T_{2}^{-k}(y), T_{2}\right) \subseteq T_{2}^{-k}(y)$ be a maximal $\left(n, \epsilon, T_{2}^{-k}(y), T_{2}\right)$-separated set for $T_{2}^{-k}(y)$, i.e.,

$$
\operatorname{card}\left(E\left(n, \epsilon, T_{2}^{-k}(y), T_{2}\right)\right)=r\left(n, \epsilon, T_{2}^{-k}(y), T_{2}\right) .
$$


Let $G \subseteq X$ be a set by taking $x^{\prime} \in \pi^{-1}\left(y^{\prime}\right)$ for each $y^{\prime} \in E\left(n, \epsilon, T_{2}^{-k}(y), T_{2}\right)$. Then $\operatorname{card}(G) \geq \operatorname{card}\left(E\left(n, \epsilon, T_{2}^{-k}(y), T_{2}\right)\right)$. For the above $y$, set $x \in \pi^{-1}(y)$. Since for all $x^{\prime} \in G$, there exists only $y^{\prime} \in E\left(n, \epsilon, T_{2}^{-k}(y), T_{2}\right)$ such that $x^{\prime} \in \pi^{-1}\left(y^{\prime}\right)$. Hence, $y=T_{2}^{k}\left(y^{\prime}\right)=$ $T_{2}^{k}\left(\pi\left(x^{\prime}\right)\right)=\pi\left(T_{1}^{k}\left(x^{\prime}\right)\right)$, further, $T_{1}^{k}\left(x^{\prime}\right)=x$, that is, $x^{\prime} \in T_{1}^{-k}(x)$, which implies that $G \subseteq T_{1}^{-k}(x)$. We claim that $G$ is an $\left(n, \delta, T_{1}^{-k}(x), T_{1}\right)$-separated set for $T_{1}^{-k}(x)$. In fact, for any $x_{1}, x_{2} \in G, \pi\left(x_{1}\right), \pi\left(x_{2}\right) \in E\left(n, \epsilon, T_{2}^{-k}(y), T_{2}\right)$. Thus

$$
d_{n}^{\prime}\left(\pi\left(x_{1}\right), \pi\left(x_{2}\right)\right):=\max _{0 \leq i<n}\left\{d^{\prime}\left(T_{2}^{i} \pi\left(x_{1}\right), T_{2}^{i} \pi\left(x_{2}\right)\right)\right\} \geq \epsilon
$$

that is, there exists $0 \leq i_{0}<n$ such that $d^{\prime}\left(T_{2}^{i_{0}} \pi\left(x_{1}\right), T_{2}^{i_{0}} \pi\left(x_{2}\right)\right) \geq \epsilon$. Since $\pi \circ T_{1}^{i_{0}}=T_{2}^{i_{0}} \circ \pi$, then $d\left(T_{1}^{i_{0}}\left(x_{1}\right), T_{1}^{i_{0}}\left(x_{2}\right)\right) \geq \delta$ by the uniform continuity of $\pi$. Thus

$$
d_{n}\left(x_{1}, x_{2}\right):=\max _{0 \leq i<n}\left\{d\left(T_{1}^{i}\left(x_{1}\right), T_{1}^{i}\left(x_{2}\right)\right)\right\} \geq \delta .
$$

Therefore, $r\left(n, \delta, T_{1}^{-k}(x), T_{1}\right) \geq \operatorname{card}(G) \geq \operatorname{card}\left(E\left(n, \epsilon, T_{2}^{-k}(y), T_{2}\right)\right)=r\left(n, \epsilon, T_{2}^{-k}(y), T_{2}\right)$, that is, $r\left(n, \delta, T_{1}^{-k}(x), T_{1}\right) \geq r\left(n, \epsilon, T_{2}^{-k}(y), T_{2}\right)$. Furthermore, we have

$$
\begin{aligned}
& \lim _{\delta \rightarrow 0} \limsup _{n \rightarrow \infty} \frac{1}{n^{s}} \log \sup _{x \in X, k \geq n} r\left(n, \delta, T_{1}^{-k}(x), T_{1}\right) \\
\geq & \lim _{\epsilon \rightarrow 0} \limsup _{n \rightarrow \infty} \frac{1}{n^{s}} \log \sup _{y \in Y, k \geq n} r\left(n, \epsilon, T_{2}^{-k}(y), T_{2}\right) .
\end{aligned}
$$

This shows that $h_{\text {pre }}\left(s, T_{1}\right) \geq h_{\text {pre }}\left(s, T_{2}\right)$ for all $s>0$. Since $\pi: X \rightarrow Y$ is a conjugate map, then $\pi^{-1}: Y \rightarrow X$ is also a conjugate map for $\left(Y, T_{2}\right)$ and $\left(X, T_{1}\right)$. Similarly, we have $h_{\text {pre }}\left(s, T_{2}\right) \geq h_{\text {pre }}\left(s, T_{1}\right)$. Therefore, $h_{\text {pre }}\left(s, T_{1}\right)=h_{\text {pre }}\left(s, T_{2}\right)$.

Let $\left(X, d_{1}, T_{1}\right)$ and $\left(Y, d_{2}, T_{2}\right)$ be two topological dynamical systems. For the product space $X \times Y$, define a map $T_{1} \times T_{2}: X \times Y \rightarrow X \times Y$ by $\left(T_{1} \times T_{2}\right)(x, y)=\left(T_{1}(x), T_{2}(y)\right)$. This map $T_{1} \times T_{2}$ is continuous and $\left(X \times Y, T_{1} \times T_{2}\right)$ forms a topological dynamical system. The metric $d$ on $X \times Y$ is given by

$$
d\left(\left(x_{1}, y_{1}\right),\left(x_{2}, y_{2}\right)\right)=\max \left\{d_{1}\left(x_{1}, x_{2}\right), d_{2}\left(y_{1}, y_{2}\right)\right\} \quad \text { for any }\left(x_{1}, y_{1}\right),\left(x_{2}, y_{2}\right) \in X \times Y \text {. }
$$

Theorem 3.5. Let $\left(X, d_{1}, T_{1}\right)$ and $\left(Y, d_{2}, T_{2}\right)$ be two topological dynamical systems. Then

$$
h_{\text {pre }}\left(s, T_{1} \times T_{2}\right)=h_{\text {pre }}\left(s, T_{1}\right)+h_{\text {pre }}\left(s, T_{2}\right) .
$$

Proof. Let $\epsilon>0, n \in \mathbb{N}$ and $k \geq n$. Since balls in the product metric $d$ are products of balls on $X$ and $Y$, the same is true for balls in the metric $d_{n}$. Hence, for $(x, y) \in X \times Y$,

$$
s\left(n, \epsilon,\left(T_{1} \times T_{2}\right)^{-k}(x, y), T_{1} \times T_{2}\right) \leq s\left(n, \epsilon, T_{1}^{-k}(x), T_{1}\right) \cdot s\left(n, \epsilon, T_{2}^{-k}(y), T_{2}\right),
$$

which implies that $h_{\text {pre }}\left(s, T_{1} \times T_{2}\right) \leq h_{\text {pre }}\left(s, T_{1}\right)+h_{\text {pre }}\left(s, T_{2}\right)$ for any $s>0$. On the other hand, the product of any $\left(n, \epsilon, T_{1}^{-k}(x), T_{1}\right)$-separated set in $T_{1}^{-k}(x)$ for $T_{1}$ and any 
$\left(n, \epsilon, T_{2}^{-k}(y), T_{2}\right)$-separated set in $T_{2}^{-k}(y)$ for $T_{2}$ is an $\left(n, \epsilon, T_{1}^{-k} \times T_{2}^{-k}(x, y), T_{1} \times T_{2}\right)$ separated set in $T_{1}^{-k}(x) \times T_{2}^{-k}(y)$ for $T_{1} \times T_{2}$. Hence,

$$
r\left(n, \epsilon,\left(T_{1} \times T_{2}\right)^{-k}(x, y), T_{1} \times T_{2}\right) \geq r\left(n, \epsilon, T_{1}^{-k}(x), T_{1}\right) \cdot r\left(n, \epsilon, T_{2}^{-k}(y), T_{2}\right),
$$

which implies that $h_{\text {pre }}\left(s, T_{1} \times T_{2}\right) \geq h_{\text {pre }}\left(s, T_{1}\right)+h_{\text {pre }}\left(s, T_{2}\right)$ for any $s>0$.

\section{Acknowledgments}

The authors are grateful to the anonymous referees for their comments, which helped to improve the paper. The first author was supported by the Foundation in higher education institutions of Henan Province, China (No. 19A110030); the National Natural Science Foundation of China (No. 11401363); the Foundation for the Training of Young Key Teachers in Colleges and Universities in Henan Province, China (No. 2018GGJS134). The second author was supported by the National Natural Science Foundation of China (No. 11801193). The third author was supported by the National Natural Science Foundation of China (No. 11971236); China Postdoctoral Science Foundation (No. 2016M591873), and China Postdoctoral Science Special Foundation (No. 2017T100384). The work was also funded by the Priority Academic Program Development of Jiangsu Higher Education Institutions. We would like to express our gratitude to Tianyuan Mathematical Center in Southwest China (11826102), Sichuan University and Southwest Jiaotong University for their support and hospitality.

\section{References}

[1] R. L. Adler, A. G. Konheim and M. H. McAndrew, Topological entropy, Trans. Amer. Math. Soc. 114 (1965), 309-319.

[2] L. S. Block and W. A. Coppel, Dynamics in One Dimension, Lecture Notes in Mathematics 1513, Springer-Verlag, Berlin, 1992.

[3] R. Bowen, Topological entropy and axiom A, in: 1970 Global Analysis (Proc. Sympos. Pure Math., Vol. XIV, Berkeley, Calif., 1968), 23-41, Amer. Math. Soc., Providence, R.I.

[4] _ Entropy for group endomorphisms and homogeneous spaces, Trans. Amer. Math. Soc. 153 (1971), 401-414.

[5] R. Bowen and P. Walters, Expansive one-parameter flows, J. Differential Equations 12 (1972), 180-193. 
[6] M. de Carvalho, Entropy dimension of dynamical systems, Portugal. Math. 54 (1997), no. $1,19-40$.

[7] W.-C. Cheng and B. Li, Zero entropy systems, J. Stat. Phys. 140 (2010), no. 5, 1006-1021.

[8] _ , Topological pressure dimension, Chaos Solitons Fractals 53 (2013), 10-17.

[9] W.-C. Cheng and S. E. Newhouse, Pre-image entropy, Ergodic Theory Dynam. Systems 25 (2005), no. 4, 1091-1113.

[10] I. P. Cornfeld, S. V. Fomin and Ya. G. Sinaŭ, Ergodic Theory, Grundlehren der Mathematischen Wissenschaften 245, Springer-Verlag, New York, 1982.

[11] D. Dou, W. Huang and K. K. Park, Entropy dimension of topological dynamical systems, Trans. Amer. Math. Soc. 363 (2011), no. 2, 659-680.

[12] Entropy dimension of measure preserving systems, Trans. Amer. Math. Soc. 371 (2019), no. 10, 7029-7065.

[13] S. Ferenczi and K. K. Park, Entropy dimensions and a class of constructive examples, Discrete Contin. Dyn. Syst. 17 (2007), no. 1, 133-141.

[14] W. Huang, K. K. Park and X. Ye, Topological disjointness from entropy zero systems, Bull. Soc. Math. France 135 (2007), no. 2, 259-282.

[15] W. Huang and Y. Yi, A local variational principle of pressure and its applications to equilibrium states, Israel J. Math. 161 (2007), 29-74.

[16] M. Hurley, On topological entropy of maps, Ergodic Theory Dynam. Systems 15 (1995), no. 3, 557-568.

[17] H. B. Keynes and M. Sears, Real-expansive flows and topological dimension, Ergodic Theory Dynam. Systems 1 (1981), no. 2, 179-195.

[18] A. N. Kolmogorov and V. M. Tihomirov, $\varepsilon$-entropy and $\varepsilon$-capacity of sets in functional space, Amer. Math. Soc. Transl. (2) 17 (1961), 277-364.

[19] R. Kuang, W.-C. Cheng and B. Li, Fractal entropy of nonautonomous systems, Pacific J. Math. 262 (2013), no. 2, 421-436.

[20] R. Kuang, W.-C. Cheng, D. Ma and B. Li, Different forms of entropy dimension for zero entropy systems, Dyn. Syst. 29 (2014), no. 2, 239-254. 
[21] R. Langevin and F. Przytycki, Entropie de l'image inverse d'une application, Bull. Soc. Math. France 120 (1992), no. 2, 237-250.

[22] Z. Li, W. Wu and Y. Zhu, Preimage pressure, stable pressure and equilibrium states, J. Differential Equations 269 (2020), no. 7, 6311-6342.

[23] D. Ma, R. Kuang and B. Li, Topological entropy dimension for noncompact sets, Dyn. Syst. 27 (2012), no. 3, 303-316.

[24] D. Ma and M. Wu, Topological pressure and topological entropy of a semigroup of maps, Discrete Contin. Dyn. Syst. 31 (2011), no. 2, 545-557.

[25] E. Mihailescu, Metric properties of some fractal sets and applications of inverse pressure, Math. Proc. Cambridge Philos. Soc. 148 (2010), no. 3, 553-572.

[26] E. Mihailescu and M. Urbański, Inverse topological pressure with applications to holomorphic dynamics of several complex variables, Commun. Contemp. Math. 6 (2004), no. 4, 653-679.

[27] _ Inverse pressure estimates and the independence of stable dimension for noninvertible maps, Canad. J. Math. 60 (2008), no. 3, 658-684.

[28] M. Misiurewicz and J. Smítal, Smooth chaotic maps with zero topological entropy, Ergodic Theory Dynam. Systems 8 (1998), no. 3, 421-424.

[29] Z. Nitecki, Topological entropy and the preimage structure of maps, Real Anal. Exchange 29 (2003/04), no. 1, 9-41.

[30] Z. Nitecki and F. Przytycki, Preimage entropy for mappings, Internat. J. Bifur. Chaos Appl. Sci. Engrg. 9 (1999), no. 9, 1815-1843.

[31] K. K. Park, On directional entropy functions, Israel J. Math. 113 (1999), 243-267.

[32] C. Robinson, Dynamical Systems: Stability, symbolic dynamics, and chaos, Second edition, Studies in Advanced Mathematics, CRC Press, Boca Raton, FL, 1999.

[33] R. F. Thomas, Some fundamental properties of continuous functions and topological entropy, Pacific J. Math. 141 (1990), no. 2, 391-400.

[34] P. Walters, A variational principle for the pressure of continuous transformations, Amer. J. Math. 97 (1975), no. 4, 937-971.

[35] _ An Introduction to Ergodic Theory, Graduate Texts in Mathematics 79, Springer-Verlag, Berlin, 1982. 
Lei Liu

School of Mathematical Sciences and Institute of Mathematics, Nanjing Normal University, Nanjing 210023, Jiangsu, China

and

School of Mathematics and Statistics, Shangqiu Normal University, Shangqiu 476000, Henan, China

E-mail address: mathliulei@163.com

Xiaomin Zhou

School of Mathematics and Statistics, Huazhong University of Science and Technology, Wuhan, Hubei 430074, China

E-mail address: zxm12@mail.ustc.edu.cn

Xiaoyao Zhou

School of Mathematical Sciences and Institute of Mathematics, Nanjing Normal University, Nanjing 210023, Jiangsu, China

E-mail address: zhouxiaoyaodeyouxian@126.com 